
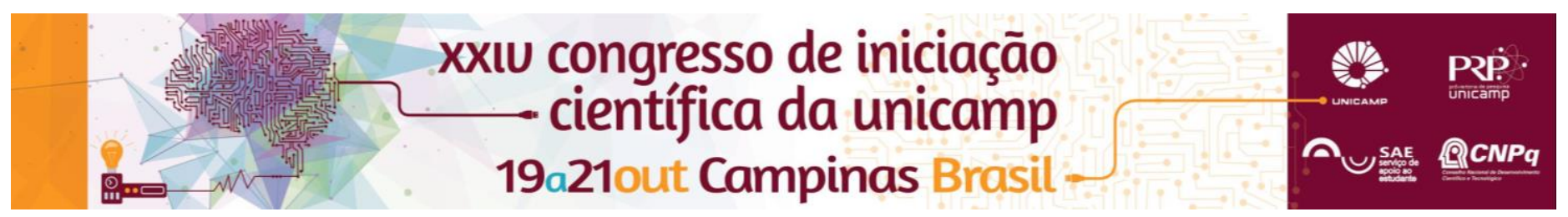

\title{
Simetrias em Física e Teorema de Noether
}

\section{Guilherme Germano Buchmeier*}

\section{Resumo}

Este projeto tem como objetivo o estudo do movimento de sistemas mecânicos contínuos, através da teoria clássica de campos. A teoria estuda o movimento utilizando o conceito de campo, uma grandeza contínua definida para cada ponto do espaço e do tempo. Com isso, é possível desenvolver o conceito de simetria e, através do teorema de Noether e da formulação Lagrangiana, nasce uma guia, na qual é possível construir as teorias de física de partículas.

\section{Palavras-chave:}

Teorema de Noether, leis de conservação, simetrias de gauge

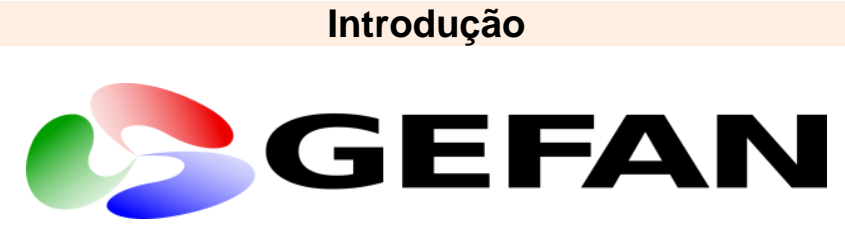

Campo designa a atribuição de uma quantidade a cada ponto existente no espaço. Para se realizar o estudo do movimento de sistemas dinâmicos, pode-se utilizar a teoria clássica de campos para desenvolver uma generalização (através do formalismo Lagrangiano) das equações dos sistemas dinâmicos em sistemas contínuos. Tal generalização permite a atribuição de equaçõs mais requintadas e completas para um sistema complexo.

Uma vez que se tenha em mãos o auxílio da teoria de campos, pode-se tratar relativisticamente o sistema em estudo, colocando em pé de igualdade quantidades como o tempo e o espaço.

O projeto visa a compreensão do formalismo Lagrangiano, formulando o teorema de Noether para que sistemas genéricos possam ser analisados, encontrando suas simetrias e cargas conservadas.

\section{Resultados e Discussão}

O princípio de Hamilton, também conhecido como princípio da mínima ação, que descreve o movimento de um sistema mecânico monogênico ${ }^{1}$ é o conceito chave fundamental para que fosse desenvolvida a teoria clássica de campos. Minimizando a ação de um sistema, foi possível chegar nas equações de EulerLagrange, as quais descrevem o movimento do sistema em questão.

Com essa ferramenta em mãos, tomamos o exemplo de uma corda oscilante, fizemos a análise discreta de cada um de seus pontos e por fim generalizamos para um sistema contínuo, tomando o limite quando essas particulas discretas formam um continuo.

Através do modelo obtido, mais uma generalização foi feita, de modo a tratar sistemas com as três coordenadas espaciais (ao invés de uma) e uma temporal. Para que pudesse haver o tratamento em pé de igualdade das quantidades espaciais e temporal, foi introduzido o conceito de quadrivetor ${ }^{2}$. A notação que passamos a utilizar com esses ajustes também foi modificada para manter a simplicidade. Com isso, foi estudado o conceito de tensor, para utiliza-lo no desenvolvimento da teoria como nova notação.
Seguindo em frente, aplicamos o princípio de Hamilton em um sistema genérico onde estariam presentes tanto a variação infinitesimal do campo quanto das coordenadas, resultando no primeiro teorema de Noether3.

O Teorema diz que cada transformação que deixa a ação invariante, existe uma combinação correspondente dos campos e suas derivadas que farão o mesmo. Desse modo, foi possível o estudo de simetrias de translação e rotação, utilizando a corrente de Noether para obter a carga conservada em cada caso.

Por fim, o campo eletromagnético foi estudado, como aplicação desse formalismo. Foram calculadas as cargas conservadas por translação, rotação e transformações de Lorentz para esse campo, mostrando a compatibilidade com os resultados esperados pelo eletromagnetismo conhecido como as equacões de Maxwell.

\section{Conclusões}

Com esse projeto, foi possível desenvolver um formalismo capaz de generalizar equações de movimento, introduzir o conceito de simetrias através da dedução do teorema de Noether e analisar as equações do eletromagnetismo de Maxwell através desse formalismo, verificando as quantidades conservadas em cada simetria. Nesse processo, foi estudado características de tensores, uma vez que são essenciais para a formulação de campos contínuos. A teoria aqui desenvolvida poderia ser extendida para análise de campos de Dirac e formulação de teorias no campo da física de partículas.

\section{Agradecimentos}

Agradeço à Unicamp e ao meu orientador por me fornecerem a oportunidade de trabalhar nesta iniciação científica, a qual coloborou com meu aprendizado extraclasse e pessoal e também, gostaria de deixar meus agradecimentos ao PIBIC, que atuou como minha Instituição de fomento.

\footnotetext{
${ }^{1}$ Herbert Goldstein. Classical mechanics. Addison-Wesley, 3rd edition, 2001 D.J.

2 David J. Griffiths. Introduction to Electrodynamics. Prentice Hall, 3rd edition, 1999.

3 R Aldrovandi and J Pereira. Classical Fields. Instituto de Física Teórica, Universidade Estadual Paulista, 1997. URL: http://www.ift.unesp.br/users/jpereira/ClassiFields.pdf
} 